\title{
Examples of Solvable Lie Foliation
}

\author{
Ameth Ndiaye \\ Correspondence: University Cheikh Anta Diop, Dakar, FASTEF. B.P. 5036, Dakar-Fann, Sénégal. \\ E-mail: ameth1.ndiaye@ucad.edu.sn
}

Received: October 23, 2018 Accepted: November 9, 2018 Online Published: December 24, 2018

doi:10.5539/jmr.v11n1p13 URL: https://doi.org/10.5539/jmr.v11n1p13

\begin{abstract}
We give here some examples of solvable Lie foliation with some interesting properties on compact manifolds. Every manifold in this paper is compact and our Lie group is simply connected.
\end{abstract}

Keywords: foliation, Lie group, solvable Lie foliation

\section{Introduction}

Let $G$ be a Lie group. A foliation on a compact differential manifold $V$ is said to be Lie foliation if it is defined by submersions from open subsets of $V$ to $G$. Lie foliations have been studied by several authors (see Dathe, H (2000), Diallo, A. S. and Ndiaye, A. (2018)...). An important class of Lie foliation given by E. Ghys (1988) is the Lie foliation, which is called homogeneous Lie foliation, obtained in the following way: $G$ and $H$ two connected, simply connected, Lie groups, $\Gamma$ a cocompact discrete subgroup of $H$, and $\varphi$ a surjective morphism of Lie groups of $H$ in $G$; the classes on the left of $H$ modulo $\operatorname{ker} \varphi$ reprojected on $H / \Gamma$ are the leaves of a $G$-foliation of $H / \Gamma$ whose holonomy morphism is the restriction of $\varphi$ to $\Gamma$ and the developing application is $\varphi$.

Else on the other hand, if $M$ and $V$ are two compact manifolds, $\mathcal{F}$ a Lie $G$ - foliation on $M$ and $f: V \longrightarrow M$ a transverse differentiable application to $\mathcal{F}$; the inverse images of leaves of $\mathcal{F}$ define a new Lie $G$-foliation $\tilde{\mathcal{F}}$ on $V$. We say that $\tilde{\mathcal{F}}$ is an inverse image of $\mathcal{F}$, in this case the holonomy group of $\tilde{\mathcal{F}}$ is a subgroup of that of $\mathcal{F}$. The naturel question is in which condition a Lie foliation can be reduced, using naturals operations, to a homogeneous Lie foliation.In the case where the Lie group is nilpotent Haefliger (1984) has shown that every nilpotent Lie foliation on compact manifolds is an inverse image of a homogeneous Lie foliation. If the Lie group is solvable G. Meigniez (1995) gives an example of Lie foliation which is not an inverse image of a homogeneous Lie foliation. The main purpose of this article is to build foliations that are not inverse image of a homogeneous Lie foliation.

\section{Preliminaries}

Let $V$ be a compact connected manifold and $G$ a Lie group (simply connected). A Lie $G$-foliation of $V$ is the given of a pair $(U ; f)$, where $U$ is an open subset of $V$ and $f: U \longrightarrow G$ a submersion, having the following properties:

i) $U$ is an open subset of $V$;

ii) for all $(U ; f)$ and $(W ; h)$ in $\mathcal{F}$, it exists $g \in G$ such as $f(x)=h(x) g$ for any $x \in U \cap W$.

In particular, the level surfaces of submersions $f$, for $(U ; f) \in \mathcal{F}$ form a foliation of $V$. To avoid ambiguities, it will be assumed that $\mathcal{F}$ is maximal in the following sense: if $U$ is an open subset of $V$ and $f: U \longrightarrow G$ a submersion, if, for any $(W ; h) \in \mathcal{F}$, it exists $g \in G$ with $f=h . g$, then $(U ; f) \in \mathcal{F}$. Let $V$ be equipped with a Lie $G$-foliation and $\mathcal{G}$ be the Lie algebra of $G$ identified with Lie algebra of right invariant vector fields of $G$. Let $x \in V$ and $(U ; f) \in \mathcal{F}$ with $x \in U$. We denote

$$
\omega_{x}=d f_{x} \in T_{x}^{*} V \otimes \mathcal{G}
$$

it is independant to $x$. This 1 -form satisfy

$$
d \omega+\frac{1}{2}[\omega, \omega]=0
$$

where $[\omega, \omega]$ is the 2 -form given by

$$
\left.[\omega, \omega]_{x}(u, v)=\left[\omega_{x}(u), \omega_{x}(v)\right]\right)
$$

for all $x \in V, u, v \in T_{x} V$.

Conversely, a section of the bundle $T_{x}^{*} V \otimes \mathcal{G}$ everywhere surjective and satisfying this condition below defines a Lie $G$ foliation. In particular the space $\mathcal{F}(G, V)$ of Lie $G$-foliations of $V$ identified with a subset of space of sections $T_{x}^{*} V \otimes \mathcal{G}$ which is locally closed for the topology of uniform convergency; it is equipped with the topology induced by it. 
Let $\tilde{V}$ be the universal covering of $V, \tilde{\mathcal{F}}$ the lifted foliation of a $G$-foliation $\mathcal{F}$ and $\tilde{\omega}$ the associated 1-form. Let's fix a point $x_{0}$ in $V$ and $\tilde{x_{0}}$ its lifted, and note $\Gamma$ the fundamental group of $V$ in $x_{0}$, which acts naturally on $\tilde{V}$. Then it exists a submersion $D: \tilde{V} \longrightarrow G$ with $D\left(\tilde{x_{0}}\right)=e$ and a morphism $h: \Gamma \longrightarrow G$ such as $\tilde{\omega}=d D$ and, for all $x \in \tilde{V}$ and for all $\gamma \in \Gamma$, we have

$$
D(\gamma \cdot x)=D(x) \cdot h(\gamma)^{-1} .
$$

We call $D$ the developing map of $\mathcal{F}$ and $h$ the holonomy morphism. Conversely, the given of pair $(D ; h)$ where $D: \tilde{V} \longrightarrow G$ is a submersion and $h: \Gamma \longrightarrow G$ a morphism with the same properties below, defines a Lie $G$-foliation in $V$. Since the group $\Gamma$ is of finite type, the set of homomorphisms of $\Gamma$ into $G$ has naturel topology locally compact. The map associates a foliation with its holonomy is continued for this topology. All these notions and results are due to E. Fedida (1971 \& 1973).

\section{Solvable Lie Foliation}

A Lie $G$-foliation is solvable if the transverse group $G$ is solvable.

We say that a foliation $\mathcal{F}$ is classifying if the holonomy fiber of each leaf is contractile. For a Lie foliation, these amounts to say that the leaves themselves are contractiles since they are without holonomy. Still according to Haefliger (1984) if $\mathcal{F}$ is a classifying Lie $G$-foliation on a compact manifold, any other Lie $G$-foliation whose holonomy is a subgroup of that of $\mathcal{F}$ is an inverse image of $\mathcal{F}$. We have the following theorem and proposition due to G. Meigniez (1995).

Theorem 1 Let $G$ be a solvable Lie group and $\Gamma$ a subgroup of $G$ of finite type. If $\Gamma$ contains a subgroup that is polycyclic and uniform, then $\Gamma$ is a holonomy group of $G$-foliation.

Proposition 2 Let $G$ be a solvable Lie group and $\Gamma$ a subgroup of $G$ of finite type and uniform. If $\Gamma$ is polycyclic and dense then $\Gamma$ is virtually holonomy group of homogenius Lie foliation.

Recall that a Lie group $G$ is polycyclic if it has a sequence of cyclic quotient composition. If $G$ is solvable and if it is of finite type with $\gamma_{1}, \ldots, \gamma_{n}$ as the generating part, then $G$ is polycyclic if and only if the zeros of $\gamma_{i}$ are algebraic units. The zeros of an element of $G$ are the eigenvalues of its adjoint. An algebraic unit is a non-zero complex number that is an algebraic integer on $\mathbb{Z}$ as well as its inverse.

So to do solvable Lie foliations which are not homogeneous, just build uniform subgroups of finite type non-polycyclics but which contain polycyclic uniform subgroups. That's what G. Meigniez (1995) does in the following example:

Example 3 We consider in GA (affine group of $\mathbb{R}$ ) the subgroup generated by $x \longmapsto \lambda x$ and $x \longmapsto \mu x+1, \lambda, \mu \neq 0$, $\lambda, \mu>0$. We suppose that $\lambda$ is algebraic unit of degree 2 and $\mu$ a real which is not algebraic unit.

So $\Gamma$ is polycyclic because it has zeros which are not algebraic units. But $\Gamma$ contains the subgroup

$$
\Gamma^{\prime}=<\lambda x, x+(\lambda-1)(\mu-1)>
$$

which is at once polycyclic and uniform, so $\Gamma$ is holonomy group of a Lie GA-foliation on a compact manifold $V$ of dimension $n$.

Since $\Gamma$ is not polycyclic then this foliation is not inverse image of homogeneous foliation.

Using this method we show

Theorem 4 There is a compact manifold with boundary, of dimension $2 n+1$ that carries a Lie GA-foliation which is not inverse image of homogeneous Lie foliation, where GA is the affine group.

Proof. Let $A \in G l_{n}(\mathbb{R})$ be a matrix

and let $G_{A}^{n+1}$ be the Lie group given by the composition law on $\mathbb{R}^{n+1}$ defined by

$$
\left(t, x_{1}, \ldots, x_{n}\right) .\left(t^{\prime}, x_{1}^{\prime}, \ldots, x_{n}^{\prime}\right)=\left(t+t^{\prime}, A^{t}\left(x_{1}^{\prime}, \ldots, x_{n}^{\prime}\right)+\left(x_{1}, \ldots x_{n}\right)\right)
$$

The Lie group $G_{A}^{n+1}$ is solvable and contain a lattice

$$
\Gamma_{A}=\left\{\left(t, x_{1}, \ldots, x_{n}\right) \in G_{A}^{n+1}: t, x_{1}, \ldots, x_{n} \in \mathbb{Z}\right\} .
$$

Let $\lambda>1$ be the eigen value of $A, v$ the associate eigen vector. Let $p$ be the proper covector of $A$ associate to $\lambda$ $\left(p: \mathbb{R}^{n} \longrightarrow \mathbb{R}\right.$ is a non vanish linear form such that $\left.p \circ A=\lambda p\right)$.

The homomorphism

$$
\begin{aligned}
\pi: G_{A}^{n+1} & \longrightarrow G A \\
\left(t, x_{1}, \ldots, x_{n}\right) & \longmapsto\left(\lambda^{t}, p\left(x_{1}, \ldots, x_{n}\right)\right)
\end{aligned}
$$


defines on $V_{A}=G_{A}^{n+1} / \Gamma_{A}$ a homogeneous Lie $G A$-foliation $\mathcal{F}$ with holonomy group $\Gamma=<(\lambda, 0) ;(1,1)>\subset G A$.

Let $a^{\prime} \in \mathbb{R}^{*}$ which is not algebraic unit and let $\Gamma^{\prime}=<\Gamma ;\left(a^{\prime}, 0\right)>$ a subgroup of $G A$.

We show that $\Gamma^{\prime}$ is the holonomy group of a Lie foliation on a manifold with boundary of dimension $2 n+1$.

Let $\delta$ be the subgroup of $\Gamma^{\prime}$ given by $\delta=<\Gamma ;\left(a^{\prime}, 0\right) \Gamma\left(a^{\prime}, 0\right)^{-1}>$. Since $\delta$ is uniform and polycyclic, then $\Gamma^{\prime}$ is holonomy group of Lie $G A$-foliation.

Now we construct the manifold supported this foliation.

Let $B$ be the matrice of $G l_{2 n}(\mathbb{R})$ given by $\left(\begin{array}{cc}A & 0 \\ 0 & A\end{array}\right)$ and the manifold $H_{B}=\mathbb{R} \times_{B} \mathbb{R}^{2 n}$ with the composition law

$$
\left(t, x_{1}, \ldots, x_{2 n}\right) \cdot\left(t^{\prime}, x_{1}^{\prime}, \ldots, x_{2 n}^{\prime}\right)=\left(t+t^{\prime}, B^{t}\left(x_{1}^{\prime}, \ldots, x_{2 n}^{\prime}\right)+\left(x_{1}, \ldots x_{2 n}\right)\right)
$$

and $\Gamma_{B}$ the lattice of integer of $H_{B}$. With this composition law, $H_{B}$ is solvable Lie group. The morphism

$$
\begin{aligned}
\phi: H_{B} & \longrightarrow G A \\
\left(t, x_{1}, \ldots, x_{2 n}\right) & \longmapsto\left(\lambda^{t}, p\left(t, x_{1}, \ldots, x_{n}\right)+a^{\prime} p\left(x_{n+1}, \ldots, x_{2 n}\right)\right)
\end{aligned}
$$

defines on $V_{B}=H_{B} / \Gamma_{B}$ a homogeneous Lie $G A$-foliation $\mathcal{F}_{B}$ and its holonomy group is $\delta$ because $\delta$ is subgroup of $\Gamma^{\prime}$ polycyclic and uniform.

Since $\mathcal{F}_{B}$ is classifying and $\Gamma \subset \delta$ then, using Haefliger (1984), it exists a map

$$
f: G_{A}^{n+1} \longrightarrow H_{B}
$$

such as $f^{*} \mathcal{F}_{B}=\mathcal{F}$. We have $\phi \circ f=\pi(\pi$ is the holonomy morphism of $\mathcal{F})$.

Let's suppose

$$
f\left(t, x_{1}, \ldots, x_{n}\right)=\left(t, x_{1}, \ldots, x_{n}, 0, \ldots, 0\right)
$$

we have $f$ is invariant by the action of $\mathbb{Z} \times_{A} \mathbb{Z}^{n}$ and the action of $\mathbb{Z} \times_{B} \mathbb{Z}^{2 n}$ and it induces an embedding again denoted by $f: V_{A} \longrightarrow V_{B}$ such as $f^{*} \mathcal{F}_{B}=\mathcal{F}$. This embedding is transverse to the foliation $\mathcal{F}_{B}$ because

$$
T_{f(x)} V_{B}=T_{f(x)} \operatorname{ker} \phi+T_{x} f\left(V_{A}\right)
$$

We call $W=V_{B}-u\left(f\left(V_{A}\right)\right.$ ) (where $u\left(f\left(V_{A}\right)\right.$ ) is a small tibular neighborhood of $u\left(f\left(V_{A}\right)\right)$ ). Then $W$ is a manifold with boundary of dimension $2 n+1$ which carries a Lie $G A$-foliation $\mathcal{F}^{\prime}$ transverse to the boundary $\partial W$. The holonomy morphism $\rho^{\prime}$ of $\mathcal{F}^{\prime}$ prolongs that of $\mathcal{F}_{B}$.

Since $V_{B}$ and $V_{A}$ are tranversly orientables, the boundary $\partial W$ of $W$ is diffeomorphic to $S^{1} \times V_{A}$ so that $\pi_{1}(W)$ has a generator $\epsilon$ of more than that of $V_{B}$.

The universel covering of $W$ is $H_{B}$ and the developing map of $\mathcal{F}^{\prime}$ is $\phi$. So $\gamma \in \pi_{1}(W) \neq \epsilon, \rho^{\prime}(\gamma)=\rho(\gamma) \in \Gamma$. If we suppose $\rho(\gamma)=(p, q)$, we have

$$
\rho^{\prime}\left(\epsilon \cdot \gamma \cdot \epsilon^{-1}\right)=\rho^{\prime}(\epsilon) \cdot \rho(\gamma) \cdot \rho^{\prime}(\epsilon)^{-1}=\left(p, a^{\prime} q\right)
$$

Which implies $\rho^{\prime}(\epsilon)=\left(a^{\prime}, 0\right)$. So the holonomy group of $\mathcal{F}^{\prime}$ is $\Gamma^{\prime}$ which is not polycyclic because $a^{\prime}$ is not algebraic unit and then $\mathcal{F}^{\prime}$ is no inverse image of any homogeneous foliation.

In the second part of this paper we give an example of non-homogeneous completely sovable Lie foliation on a compact manifold.

Definition 5 A solvable Lie group $G$ with Lie algebra $\mathcal{G}$ is said completely solvable if all linear operators ad $d_{X}(X \in \mathcal{G})$ have only real eigenvalues.

Theorem 6 There is a Lie foliation (on a compact manifold) which is not homogeneous whose transverse group is completely solvable.

Proof. Let $N$ the Heisenberg group of dimension 3 and $\Gamma$ the cocompact lattice of $K=N \times N$ given by matrix of integers coefficients.

We denote by $G_{6}$ the Lie group whose Lie algebra is defined by

$$
\mathcal{G}=\left(\mathbb{R}^{4}, e_{1}, e_{2}, e_{3}, e_{4}\right)
$$

such as the non-vanish bracket are $\left[e_{4}, e_{1}\right]=e_{2}$ and $\left[e_{4}, e_{2}\right]=e_{3}$. It is easy to see that $G_{6}$ is completely solvable.

We show that the manifold $M=K / \Gamma$ can be equipped with a Lie $G_{6}$-foliation.

The group $G_{6}$ is the semi-direct product of the group $S$ isomorph to $\mathbb{R}$ with the group $V$ isomorph to $\mathbb{R}^{3}$. The group $S$ acting on $V$ by a subgroup of one parameter defined by

$$
r(t)=\exp A t
$$


(where $A$ is a square matrix of order 3 invertible and has only pure imaginary eigenvalues) and we denote by $D \subset S$ the kernel of this parameter subgroup. Since this construction then the map $\phi: S \times V \longrightarrow G_{6}$ is a diffeomorphism defined by

$$
\phi(t, v) \cdot \phi\left(t^{\prime}, v^{\prime}\right)=\phi\left(t+t^{\prime}, v+r(t) v^{\prime}\right)
$$

where $r(t)$ is the rotation of angle $2 \pi t$.

Since $\Gamma \cap[K, K]$ is a lattice of $[K, K]$ and $K /[K, K]$ is isomorph to $\mathbb{R}^{4}$, then the projection

$$
p: K \longrightarrow K /[K, K]
$$

send $\Gamma$ to a lattice of $\mathbb{R}^{4}$

It exists a surjectif morphism $\psi: K \longrightarrow S \times V$ such as $\psi(\Gamma) \subset D$.

The map $\phi \circ \psi$ is the developing map of a Lie $G_{6}$-foliation on the manifold $M$ whose holonomy morphism is the restriction of $\phi \circ \psi$ to $\Gamma$. This foliation is not homogeneous because $\phi \circ \psi$ is not a morphism of group of $K$ to $G_{6}$.

\section{Conclusion}

Thus, the aim of this work was to prove that in heigher dimension if the transverse structure is solvable it doesn't exist a Lie foliation which is inverse image of homogeneous Lie foliation. In the second part we construct a non-trivial completely solvable Lie foliation. Our perspectives are to classify the Lie foliations that are semi-simple and completely solvable.

\section{Acknowledgements}

The author would like to thank the Referee for his comments and suggestions.

All many thanks to professor Bakary Manga from University Cheikh Anta Diop of Dakar for their remarks.

\section{References}

Dathe, H. (2000). Sur l'existence des feuilletages de Lie. Afrika Mat. 11(3), 15 - 20.

Diallo, A. S., \& Ndiaye, A. (2018). On Completely Solvable Lie Foliation, Glob. J. Adv. Res. Class. Mod. Geom., 7(2), $102-106$.

Fedida, E. (1971). Sur les feuilletages de Lie, C. R. Acad. Sci. Paris (272), 999 - 1001.

Fedida, E. (1973). Feuilletages du plan, feuilletage de Lie, thése université Louis Pasteur, Strasbourg.

Ghys, E. (1988). Riemannian Foliation: Example and Problems, Appendix E of Riemannian foliations, by P. Molino, Progress in Mathematics, vol.73, Birkhäuser, Boston.

Haefliger, A. (1984). Groupoide d'holonomie et classifiants: Structures transverses des feuilletages, Astéristique, SMF 116.

Meignez, G. (1995). Feuilletages de Lie résolubles, Annales de la faculté des sciences de Toulouse, 4(4).

\section{Copyrights}

Copyright for this article is retained by the author(s), with first publication rights granted to the journal.

This is an open-access article distributed under the terms and conditions of the Creative Commons Attribution license (http://creativecommons.org/licenses/by/4.0/). 